Research Paper

\title{
Prognostic Value of XIAP Level in Patients with Various Cancers: A Systematic Review and Meta-Analysis
}

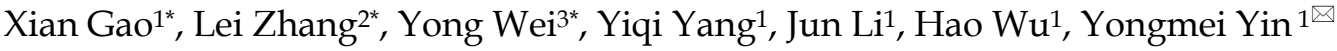 \\ 1. Department of Oncology, The First Affiliated Hospital of Nanjing Medical University, Nanjing 210029, China. \\ 2. Department of Urology, The First Affiliated Hospital of Nanjing Medical University, Nanjing, 210009, China. \\ 3. Department of Urology, Nanjing Gaochun People's Hospital, Nanjing, 211300, China \\ *The authors contributed equally to this work. \\ $\triangle$ Corresponding author: Yongmei Yin, Department of Oncology, The First Affiliated Hospital of Nanjing Medical University, No. 300 Guangzhou Road, \\ Nanjing, 210029, China. E-mail: ymyin@njmu.edu.cn; TEL: +86 18360867872 \\ (c) Ivyspring International Publisher. This is an open access article distributed under the terms of the Creative Commons Attribution (CC BY-NC) license \\ (https://creativecommons.org/licenses/by-nc/4.0/). See http://ivyspring.com/terms for full terms and conditions.
}

Received: 2018.07.01; Accepted: 2018.12.15; Published: 2019.02.26

\begin{abstract}
Background: X-linked inhibitor of apoptosis protein (XIAP) plays an important role in cancer pathogenesis, which has been found to be overexpressed in multiple human cancers and associated with survival rates. Herein, we performed a meta-analysis to explore the predictive value of XIAP level in patients with various solid tumors.

Methods: Relevant articles exploring the relationship between XIAP expression and survival of cancer patients were retrieved in PubMed, PMC, EMBASE and Web of Science published from 2001 to 2018 . The combined hazard ratios (HRs) and $95 \%$ confidence intervals $(\mathrm{Cls})$ were calculated to evaluate the significance.

Results: A total of 6554 patients from 40 articles were included in this meta-analysis. It was shown in 37 studies with 4864 cases that the over-expression of XIAP was associated with poorer overall survival (OS) (combined HR=1.61, 95\% Cl: 1.33-1.96). Meanwhile, 8 studies with 1862 cases revealed that elevated XIAP level predicted shorter disease-free survival (DFS) (HR=2.17, $95 \% \mathrm{Cl}$ : 1.03-4.59). Subgroup analyses showed that higher XIAP detection was related to worse OS in gastric cancer $(\mathrm{HR}=1.42,95 \% \mathrm{Cl}: 1.18-1.72)$ and head and neck cancer $(\mathrm{HNC})(\mathrm{HR}=2.97,95 \% \mathrm{Cl}$ : 1.97-4.47).

Conclusion: Our results suggested that elevated XIAP level seemed to represent an unfavorable prognostic factor for clinical outcomes in cancer patients. However, there were limited studies describing the association between XIAP expression and clinical prognosis in each different type of tumors. Therefore, concrete roles of XIAP in various cancers need to be further explored.
\end{abstract}

Key words: XIAP, cancer, survival, meta-analysis

\section{Introduction}

In humans, the balance between cell proliferation and cell death is essential to the homeostasis of microenvironment [1]. Programmed cell death (PCD) such as apoptosis or necroptosis performs a major mechanism for precise regulation of cell number and morphological architecture [2]. Cell apoptosis enables elimination of damaged and senescent cells, and cells that harbor too many mutations [3]. Once pro-apoptotic factors are suppressed or anti-apoptotic proteins are upregulated, normal cell turnover escapes from biological cell-death signals, promoting a tumor to thrive [4].

Inhibitors of apoptosis proteins (IAPs) are a family of proteins composed of eight members. The family has been studied extensively due to the anti-apoptotic effect as well as its over-expression potential in cancer cells. X-linked inhibitor of apoptosis protein (XIAP), also named BIRC4, is a key 
member of the IAPs. Its encoded gene has been identified mapped to the Xq25 chromosome region. Except for peripheral blood leukocytes, XIAP is almost ubiquitously expressed in all human tissues and mainly located in cytosolic compartment [5]. Structurally, XIAP has three characteristic functional domains in N-terminal known as zinc finger baculoviral IAP repeats (BIRs). The BIR domains interact with proteins that modulate NF-kB signaling or bind to active caspase- $9,-3$ and -7 , which are responsible for the caspase inhibitory function of XIAP [6-9]. Besides, the C-terminal part of XIAP carries a ubiquitin-associated (UBA) domain for ubiquitin-binding and a really interesting new gene (RING) domain with E3-ubiquitin ligase activity, which involve in the protein degradation mediated by ubiquitin-proteasome pathway [10].

As a key regulator of apoptotic cell death, XIAP has been found to be associated with tumorigenesis, angiogenesis, tumor invasion and resistance to anticancer drugs in cancer therapy [10]. It was slowly emerging as a potential therapeutic target for cancer management. Recently, many scholars have carried out series of studies to explore the expression of XIAP and its association with patient outcomes in different types of tumors, such as hepatocellular carcinoma (HCC), breast cancer, non-small cell lung cancer (NSCLC), esophageal cancer, thyroid cancer, prostate cancer [11-16]. However, these results remain controversial. Shi et al. suggested that over-expression of XIAP could be an independent prognostic factor for predicting poor survival rate in HCC patients while $\mathrm{Wu}^{\prime}$ s study indicated that low XIAP expression contributed to a worse prognosis [12, 17]. What's more, XIAP was regarded as a valuable poor prognostic marker for breast cancer according to Hussain and his colleagues, while it was found unrelated with patients' survival in the research conducted by $\mathrm{Xu}[18,19]$. Up till now, there has been no consensus on the specific role of XIAP in cancer survival. Hence, we performed a meta-analysis to illustrate the prognostic value of XIAP and aimed to provide a rationale for future clinical studies to investigate therapeutic targets for cancer.

\section{Material and Methods}

\section{Publication search and inclusion criteria}

A comprehensive literature retrieval was conducted in PubMed, PMC, EMBASE and Web of Science databases. Medical subheading (Mesh) terms relating to XIAP (e.g. "XIAP," "X-linked inhibitor of apoptosis protein," "baculoviral inhibitor of apoptosis repeat containing 4" or "BIRC4") in combination with words related to prognosis (e.g. "prognosis," "survival," "outcome" or "predict") and terms to cancer (e.g. "cancer," "tumor," "carcinoma" or "neoplasm") were used to retrieve eligible studies for our analyses until June 2018. Furthermore, the reference lists of the selected articles which may contain additional potentially relevant studies were also examined.

Articles were considered eligible if they met the following criteria: (a) studies focused on the prognostic value of XIAP expression for overall survival (OS), disease-free survival (DFS), progression-free survival (PFS) or recurrence-free survival (RFS); (b) the patients were diagnosed with solid tumors; (c) XIAP protein level was detected in tumor samples; (d) hazard ratios (HRs) and their 95\% confidence intervals (95\% CIs) for survival analysis were reported or could be computed from given data.

The exclusion criteria were: (a) abstract, case report, review, expert opinion or comment letter; (b) the patients with hematological malignancies; (c) studies which revealed duplicate data or repeated analysis; (d) non-human research.

\section{Data extraction}

Two reviewers independently extracted data from included articles under the guidelines of a critical review checklist of the Dutch Cochrane Centre proposed by Meta-analysis of Observational Studies in Epidemiology (MOOSE) [20]. For each selected publication, we recorded the following information: first author, year of publication, country of origin, total number of participants, ethnicity, cancer type, detection method, cut-off value and survival end points. For discrepancies, discussion sustained until reaching a consensus.

\section{Assessment of study quality}

The Newcastle-Ottawa Scale (NOS) criteria was used to assessed the methodological quality of the eligible researches by 2 independent reviewers [21]. The scale evaluates three perspectives of study groups: selection (0-4), comparability (0-2) and ascertainment of clinical outcomes (0-3). Total NOS scores range from 0 to 9 . Studies with scores higher than 7 represented good quality while scores lower than 4 were excluded in our meta-analysis. Disagreement on calculated scores was solved by discussion, or a third investigator was consulted.

\section{Statistical analysis}

Combined HRs and 95\% CIs were computed to reveal the associations between XIAP expression and prognosis (OS, DFS, PFS and RFS) in cancer patients. The HRs and 95\% CIs were extracted from primary articles. If the HR was not provided directly in a study, Engauge Digitizer was used to read survival 
rates from Kaplan-Meier curves. We estimated censoring using the minimum and maximum follow-up times or the reported numbers at risk and input summary statistics into the calculations spreadsheet given by Tierney to obtain the HR estimate and its variance [22].

Cochran's $Q$ test and the Higgins I-squared statistic were performed to investigate the heterogeneity of combined results. In the cases of substantial heterogeneity among studies $\left(I^{2} \geq 50 \%\right.$ or $\left.P<0.05\right)$, a random-effect model (DerSimonian-Laird method) was applied to calculate the combined HR [23]. Otherwise, a fixed-effect model (the Mantel-Haenszel method) was chosen [24]. Moreover, meta-regression and subgroup analyses were conducted to assess the possible sources of heterogeneity. In addition, sensitivity analyses were used to identify the stability of summary effect estimate. Begg's funnel plots and Egger's linear regression test were adopted to detect publication bias with $P<0.05$ considered statistically significant. All reported $p$-values are two-sided. All statistical calculations were performed by using STATA software (version 12.0, Stata Corporation, College Station, TX).

\section{Results}

\section{Study selection and characteristics}

A total of 632 potentially relevant literatures were retrieved through the initial database search. After title and abstract screening, 537 articles were excluded. Finally, the rest 95 articles were assessed in full text and 55 additional articles were excluded: 2 articles presented overlapping data, 26 articles lacked sufficient data that could not be extracted and 27 articles did not deal with prognostic information about XIAP expression. Eventually, 40 articles met the inclusion criteria were included in our meta-analysis. Selection process was summarized in Figure 1.

Basic characteristics of the 40 enrolled articles were shown in Table 1. These studies were published from 2001 to 2018, with 6554 patients in 13 countries. All studies were retrospective design and the sample size ranged from 29 to 1103 . The patients were diagnosed with various solid carcinomas, among which digestive tumors accounted for the most type, particularly gastric cancer $(n=4)$ [25-28], colorectal cancer $(n=3)$ [29-31], HCC $(n=5)$ [12, 17, 32-34], esophageal cancer $(n=2)[11,35]$, cholangiocarcinoma $(n=2)[36,37]$ and pancreatic cancer $(n=1)$ [38]. And the remaining studies included breast cancer $(\mathrm{n}=4)$ $[16,18,19,39]$, NSCLC $(n=2)$ [13, 40], head and neck cancer $(\mathrm{HNC})(\mathrm{n}=4)$ [41-44], thyroid cancer $(\mathrm{n}=4)$ $[15,45-47]$, prostate cancer $(n=2)[14,48]$, renal cancer $(n=2)[49,50]$ and other tumor types $(n=5)$ listing as: sebaceous gland carcinoma, glioblastomas, ovarian cancer, bladder cancer and malignant mesothelioma [51-55]. Most studies used immunohistochemistry (IHC) to examine the expression of XIAP in tissue specimens except for 2 studies which detected the expression of XIAP by western blot [27] and protein pathway array [49]. Overall, 55 studies included in this article: 37 studies for OS, 8 studies for DFS, 4 studies for PFS and 6 studies for RFS.

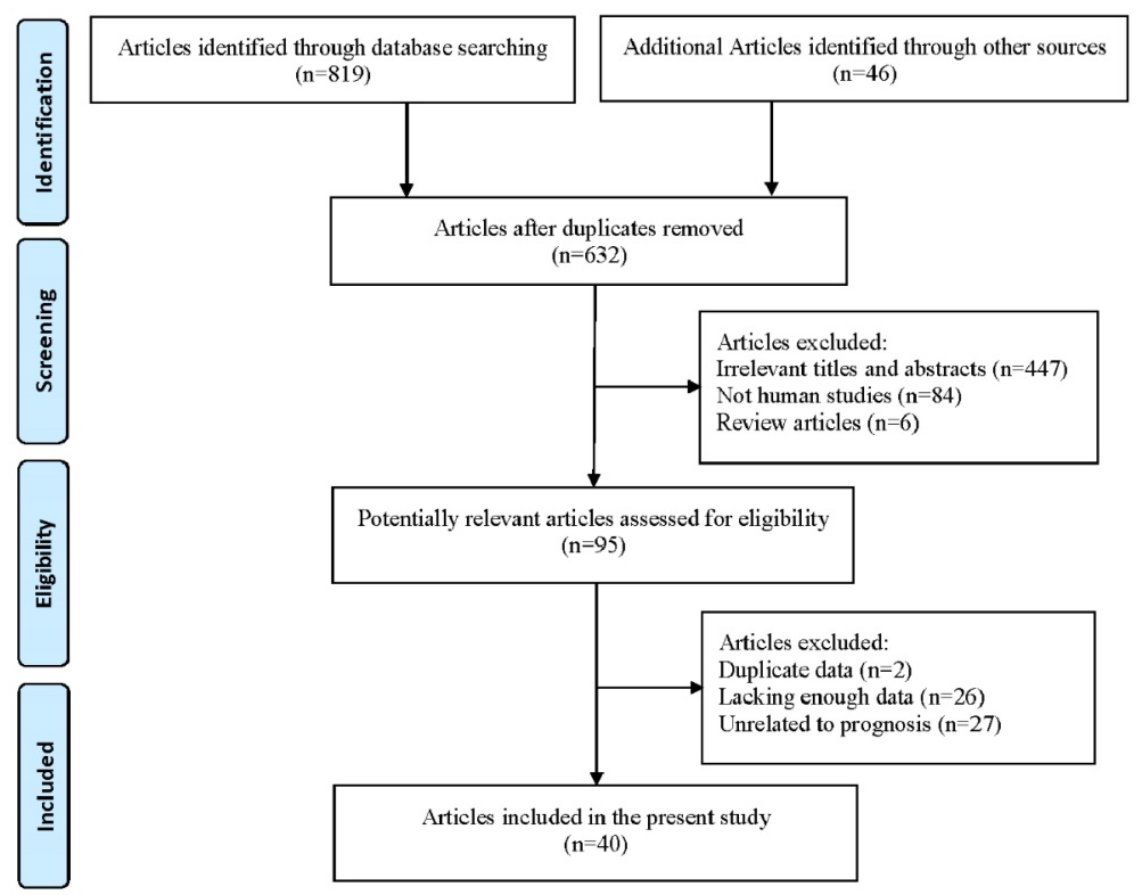

Figure 1. Flowchart for the study selection process. 
Table 1. Main characteristics of the studies included in this meta-analysis.

\begin{tabular}{|c|c|c|c|c|c|c|c|c|c|c|c|}
\hline Author & Year & Country & Samples & Ethnicity & Type & Location & Method & Cut-off level & Outcome & HR estimate & NOS \\
\hline Dizdar & 2018 & Germany & 175 & Caucasus & Esophageal & Cytoplasm & IHC & score 2 & OS & HR & 8 \\
\hline Javarai & 2017 & India & 29 & Asian & Sebaceous gland & Cytoplasm & $\mathrm{IHC}$ & score 2 & DFS & Curve & 5 \\
\hline Dizdar & 2017 & Germany & 149 & Caucasus & Gastric & Cytoplasm & IHC & score 2 & OS & HR & 8 \\
\hline Hussain & 2017 & Saudi Arabia & 964 & Caucasus & Breast & NR & $\mathrm{IHC}$ & score 85 & Os & HR & 5 \\
\hline Frohwitter & 2017 & Germany & 83 & Caucasus & Head and neck & Cytoplasm & $\mathrm{IHC}$ & $\mathrm{N} / \mathrm{P}$ & OS & Curve & 6 \\
\hline Emery & 2017 & America & 50 & Caucasus & Glioblastomas & NR & $\mathrm{IHC}$ & score 50 & OS & HR & 6 \\
\hline $\mathrm{Li}$ & 2017 & China & 70 & Asian & Hepatocellular & Cytoplasm & $\mathrm{IHC}$ & score 3 & OS & Curve & 5 \\
\hline Werner & 2017 & Germany & 34 & Caucasus & Thyroid & Cytoplasm & IHC & score 8 & OS & HR & 7 \\
\hline Dizdar & 2016 & Germany & 57 & Caucasus & Gastric & Cytoplasm & IHC & score 2 & OS & HR & 7 \\
\hline Ren & 2016 & China & 109 & Asian & Head and neck & Cytoplasm & IHC & score 2 & OS & HR & 6 \\
\hline Chen & 2016 & China & 58 & Asian & Colorectal & Cytoplasm & $\mathrm{IHC}$ & $25 \%$ PC & OS & HR & 7 \\
\hline Werner & 2016 & Germany & 74 & Caucasus & Thyroid & Cytoplasm & $\mathrm{IHC}$ & score 6 & OS & HR & 7 \\
\hline $\mathrm{He}$ & 2016 & China & 32 & Asian & Gastric & NR & Array & NR & OS/RFS & HR & 5 \\
\hline Gonzalo & 2015 & Spain & 84 & Caucasus & Prostate & Cytoplasm & $\mathrm{IHC}$ & $5 \%$ PC & PFS & HR & 5 \\
\hline Hussain & 2015 & Saudi Arabia & 1022 & Caucasus & Thyroid & NR & IHC & score 40 & DFS & Curve & 6 \\
\hline Wu & 2014 & China & 150 & Asian & Hepatocellular & Cytoplasm & $\mathrm{IHC}$ & $25 \%$ PC & OS/DFS & Curve & 7 \\
\hline Miyamoto & 2014 & Japan & 90 & Asian & Ovarian & NR & IHC & score 2 & OS/PFS & HR/Curve & 7 \\
\hline $\mathbf{X u}$ & 2014 & China & 100 & Asian & Breast & Cytoplasm/Nuclear & $\mathrm{IHC}$ & score 6 & OS/DFS & Curve & 6 \\
\hline Zhou & 2013 & China & 50 & Asian & Cholangio & Cytoplasm & $\mathrm{IHC}$ & score 4 & OS & HR & 7 \\
\hline $\mathrm{Li}$ & 2013 & China & 52 & Asian & Pancreatic & Cytoplasm & $\mathrm{IHC}$ & score 2 & OS & HR & 7 \\
\hline Zhou & 2013 & China & 78 & Asian & Esophageal & Cytoplasm & $\mathrm{IHC}$ & score 3 & OS/PFS & HR & 7 \\
\hline $\mathbf{X u}$ & 2013 & China & 50 & Asian & Cholangio & Cytoplasm & $\mathrm{IHC}$ & score 4 & OS & HR & 6 \\
\hline Yim & 2012 & Italy & 123 & Caucasus & Thyroid & NR & $\mathrm{IHC}$ & score 4 & RFS & Curve & 5 \\
\hline Che & 2012 & China & 59 & Asian & Hepatocellular & NR & IHC & score 4 & OS & Curve & 5 \\
\hline Yang & 2012 & China & 60 & Asian & Head and neck & Cytoplasm & $\mathrm{IHC}$ & $25 \%$ PC & OS & HR & 7 \\
\hline Hector & 2012 & Ireland & 224 & Caucasus & Colorectal & NR & $\mathrm{IHC}$ & score 2 & OS/RFS & HR & 7 \\
\hline Kim & 2011 & Korea & 1103 & Asian & Gastric & Cytoplasm & IHC & $10 \%$ PC & OS & HR & 6 \\
\hline Zhang & 2011 & China & 102 & Asian & Breast & Cytoplasm/Nuclear & $\mathrm{IHC}$ & score 2 & OS & HR & 7 \\
\hline Nagata & 2011 & Japan & 54 & Asian & Head and neck & Cytoplasm & IHC & score 20 & OS & HR & 7 \\
\hline Wang & 2010 & China & 42 & Asian & Breast & Cytoplasm & IHC & $50 \%$ PC & OS/DFS & HR & 8 \\
\hline Xiang & 2009 & China & 96 & Asian & Colorectal & Cytoplasm & $\mathrm{IHC}$ & score 2 & OS/DFS & HR & 7 \\
\hline Augello & 2009 & Italy & 69 & Caucasus & Hepatocellular & Cytoplasm & $\mathrm{IHC}$ & score 6 & OS & Curve & 6 \\
\hline Shi & 2008 & China & 192 & Asian & Hepatocellular & Cytoplasm & IHC & score 4 & OS/DFS & HR & 7 \\
\hline Seligson & 2007 & America & 192 & Caucasus & Prostate & Cytoplasm & $\mathrm{IHC}$ & score 1.8 & RFS & HR & 7 \\
\hline $\mathrm{Li}$ & 2007 & China & 176 & Asian & Bladder & NR & $\mathrm{IHC}$ & $\mathrm{N} / \mathrm{P}$ & DFS & HR & 6 \\
\hline Kleinberg & 2007 & Norway & 62 & Caucasus & Mesothelioma & Cytoplasm & IHC & $\mathrm{N} / \mathrm{P}$ & OS & Curve & 5 \\
\hline Mizutani & 2007 & Japan & 109 & Asian & Renal & NR & WB & 2 -fold & DFS & Curve & 6 \\
\hline Ramp & 2004 & Germany & 145 & Caucasus & Renal & Cytoplasm & IHC & NR & OS & Curve & 5 \\
\hline Ferreira & 2001 & Netherlands & 144 & Caucasus & NSCLC & Cytoplasm & IHC & $20 \%$ PC & OS & HR & 5 \\
\hline Ferreira & 2001 & Netherlands & 42 & Caucasus & NSCLC & Cytoplasm & IHC & $25 \%$ PC & OS & Curve & 6 \\
\hline
\end{tabular}

IHC: immunohistochemistry; WB: western blot; NOS: Newcastle-Ottawa quality assessment scale; NSCLC: Non-small cell lung cancer; PC: Positive cells; NR: not reported.

\section{Prognostic value of XIAP expression for patient survival}

As shown in Figure 2, the OS revealed that high level of XIAP predicted a poor outcome $(\mathrm{HR}=1.61$, 95\% CI=1.33-1.96, $P$ heterogeneity $<0.001)$. Similarly, the prognostic role of XIAP expression for DFS was investigated with a combined HR of 2.17 (95\% CI: 1.03-4.59, $P$ heterogeneity $<0.001$ ) (Figure 3). But in terms of PFS (HR=1.09, 95\% CI: 0.39-3.04, $P$ heterogeneity $=0.015)$ and RFS (HR=0.93, 95\% CI: 0.64-1.35, $P$ heterogeneity $=0.013)$, the results were considered no statistical significance.

Regarding OS, subgroup analyses by cancer type showed that XIAP expression predicted an unfavorable prognosis in gastric cancer $(\mathrm{HR}=1.42$, 95\% CI: 1.18-1.72, $P$ heterogeneity $=0.105)$ and HNC $(\mathrm{HR}=2.97,95 \%$ CI: 1.97-4.47, $P$ heterogeneity $=0.515)$. As for colorectal cancer, the combined HR was 1.75 (95\% CI: 0.99-3.11, $P$ heterogeneity $<0.001)$, indicating marginal significance of poor survival. However, no sufficient evidence was acquired to demonstrate the association between XIAP expression and clinical outcomes in hepatocellular carcinoma ( $\mathrm{HR}=2.28,95 \% \mathrm{CI}$ : 0.76-6.87, $P$ heterogeneity $<0.001)$, esophageal cancer $(\mathrm{HR}=1.77,95 \%$ CI: $0.84-3.75, P$ heterogeneity $=0.024$ ), cholangiocarcinoma $\left(\mathrm{HR}=0.98,95 \% \mathrm{CI}: 0.51-1.86, P_{\text {heterogeneity }}=0.937\right)$, breast cancer (HR=1.66, 95\% CI: 0.61-4.50, $P$ heterogeneity $=0.017)$ as well as thyroid cancer (HR=1.20, 95\% CI: 0.59-2.47, $P$ heterogeneity $=0.003$ ). Surprisingly, NSCLC patients in two studies with higher XIAP expression had better survival rates than patients expressed lower XIAP level, with the HR of 0.65 (95\% CI: 0.45-0.94, $P$ heterogeneity $=0.767$ ).

Based on the median cut-off value (IHC score=3), the cohorts were divided into the high value and low value groups. Our results indicated that the HRs were 1.87 (95\% CI: 1.18-2.95, $P$ heterogeneity $<0.001)$ and 1.49 (95\% CI: 1.12-1.97, $P$ heterogeneity $=0.001)$ for high and low 
cut-off value group, respectively. Meanwhile, we analyzed cut-off value on two more groups divided by percentage of positive staining cells, both results were considered no significance $(25 \%$ positive cells group: $\mathrm{HR}=1.48,95 \% \mathrm{CI}$ : $0.48-4.56, P$ heterogeneity $<0.001$; non-25\% positive cells group: $\mathrm{HR}=1.43,95 \% \mathrm{CI}$ : 0.60-3.41, $P$ heterogeneity $<0.001)$.

Dealing with different ethnicities, high XIAP expression was a negative prognostic marker for both Caucasian patients (HR=1.29, 95\% CI: 1.05-1.59, $P$ heterogeneity $<0.001)$ and Asian patients $(\mathrm{HR}=2.07,95 \% \mathrm{CI}$ : 1.47-2.92, $P$ heterogeneity $<0.001)$. When performing subgroup analyses stratified by number of participants (since median number was 83, studies with more than 83 participants were classified as "large" and studies with less than 83 patients were classified as "small"), we found that XIAP level predicted unfavorable survival regardless of large (HR=1.41, 95\% CI: 1.07-1.87, $\left.P_{\text {heterogeneity }}<0.001\right)$ or small sample size $(\mathrm{HR}=1.83, \quad 95 \% \quad \mathrm{CI}: 1.38-2.42, \quad P$ heterogeneity $<0.001)$. Moreover, our result demonstrated that data from multivariate analysis ( $\mathrm{HR}=1.69,95 \%$ CI: 1.35-2.13, $\left.P_{\text {heterogeneity }}<0.001\right)$ and univariate analysis $(\mathrm{HR}=1.48,95 \%$ CI: 1.01-2.17, $P$ heterogeneity $<0.001)$ both revealed unfavorable OS.

Regarding DFS, we conducted subgroup analyses by ethnicity and data resource. When different ethnicities were considered, XIAP-high expression was seemed to be a negative prognostic marker for Asian patients (HR=3.40, 95\% CI: 2.17-5.33, $P$ heterogeneity $=0.336)$, while not for Caucasian patients $(\mathrm{HR}=0.39$, 95\% CI: 0.04-3.64, $P$ heterogeneity $=0.002)$. Additionally, the studies adopted univariate analysis had an HR of 1.95 (95\% CI: 0.92-4.13, $P$ heterogeneity $=0.012$ ) and multivariate analysis showed an HR of 2.24 (95\% CI: 0.35-14.34, $P$ heterogeneity $<0.001$;).

All of the detailed results of overall analyses as well as subgroup analyses were put in Table 2.

\section{Heterogeneity, Sensitivity analysis and Publication bias}

Meta-regression was conducted with the following variables: year of publication, number of participants (large vs. small), ethnicity, cancer type, cut-off value and data resource (univariate vs. multivariate) to explore the potential source of heterogeneity among studies. The results showed that year of publication, number of participants, cancer type, cut-off value and data resource did not contribute to the source of heterogeneity significantly. However, ethnicity was an exception $(P=0.05)$ and this variable could explain $11.8 \%$ of the heterogeneity.

To evaluate sensitivity, we sequentially removed each individual study to investigate their influence on the combined results of XIAP expression for OS and
DFS (Figures 4A and 4B). The combined HRs were found to be stable, suggesting no individual study significantly affected the results.

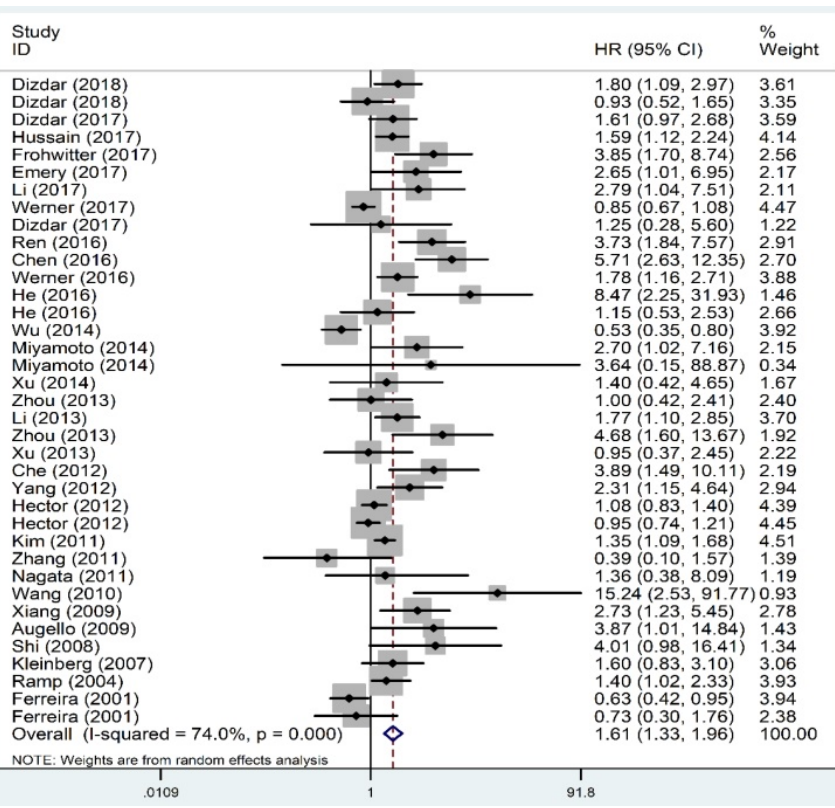

Figure 2. Forest plots of studies evaluating HRs of XIAP expression for OS of cancer patients. HR: hazard ratio; XIAP: X-linked inhibitor of apoptosis protein; OS: overall survival.

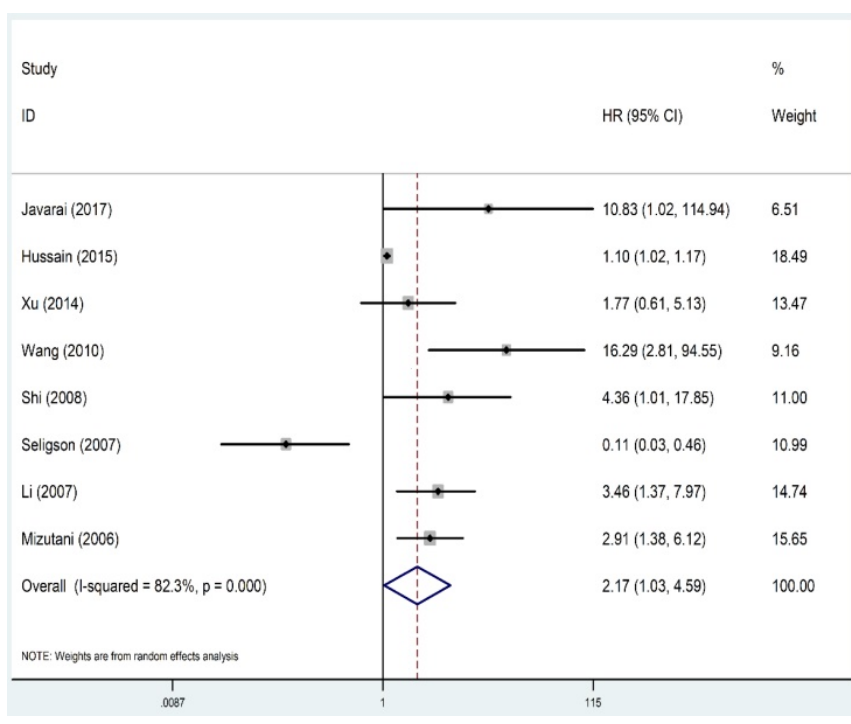

Figure 3. Forest plots of studies evaluating HRs of XIAP expression for DFS of cancer patients. HR: hazard ratio; XIAP: X-linked inhibitor of apoptosis protein; DFS: disease-free survival.

Then Begg's funnel plot and the Egger's linear regression test were used to assess publication bias. As shown in Figure 5A, for OS, the funnel plot was asymmetric with the $P=0.038$. The calculation of Egger's test was $P=0.001$, revealing the existence of bias. For further confirmation, trim and fill method was utilized and the combined HR with hypothetically non-published studies were recalculated to 
evaluate the indicative value of XIAP expression included studies evaluating the DFS outcome (Figure 6). The eventual HR was 1.27 (95\% CI: 1.05-1.55, $P$ heterogeneity $<0.001)$ which was considered suggested no publication bias existed in our analysis statistical significance. In addition, the results for the

(Figure 5B: Begg's $P=0.711$, Egger's $P=0.138$ ).

Table 2. Meta-analysis results.

\begin{tabular}{|c|c|c|c|c|c|}
\hline Outcome & Variables & Number of studies & Model & HR $(95 \% \mathrm{CI})$ & $P_{\text {heterogeneity }}$ \\
\hline \multirow[t]{27}{*}{ OS } & ALL & 37 & Random & $1.61(1.33-1.96)$ & $<0.001$ \\
\hline & Cancer type & & & & \\
\hline & Gastric & 5 & Fixed & $1.42(1.18-1.72)$ & 0.105 \\
\hline & Hepatocellular & 5 & Random & $2.28(0.76-6.87)$ & $<0.001$ \\
\hline & Colorectal & 4 & Random & $1.75(0.99-3.11)$ & $<0.001$ \\
\hline & Esophageal & 3 & Random & $1.77(0.84-3.75)$ & 0.024 \\
\hline & Cholangio & 2 & Fixed & $0.98(0.51-1.86)$ & 0.937 \\
\hline & Breast & 4 & Random & $1.66(0.61-4.50)$ & 0.017 \\
\hline & $\mathrm{HNC}$ & 4 & Fixed & $2.97(1.97-4.47)$ & 0.515 \\
\hline & Thyroid & 2 & Random & $1.20(0.59-2.47)$ & 0.003 \\
\hline & NSCLC & 2 & Fixed & $0.65(0.45-0.94)$ & 0.767 \\
\hline & Others & 6 & Fixed & $1.69(1.30-2.19)$ & 0.725 \\
\hline & Ethnicity & & & & \\
\hline & Asian & 21 & Random & $2.07(1.47-2.92)$ & $<0.001$ \\
\hline & Caucasian & 16 & Random & $1.29(1.05-1.59)$ & $<0.001$ \\
\hline & Resource & & & & \\
\hline & Univariate & 14 & Random & $1.48(1.01-2.17)$ & $<0.001$ \\
\hline & Multivariate & 23 & Random & $1.69(1.35-2.13)$ & $<0.001$ \\
\hline & Participate number & & & & \\
\hline & Large & 14 & Random & $1.41(1.07-1.87)$ & $<0.001$ \\
\hline & Small & 23 & Random & $1.83(1.38-2.42)$ & $<0.001$ \\
\hline & $\begin{array}{l}\text { Cut off level } \\
\text { score }\end{array}$ & & & & \\
\hline & score high & 10 & Random & $1.87(1.18-2.95)$ & $<0.001$ \\
\hline & score low & 12 & Random & $1.49(1.12-1.97)$ & 0.001 \\
\hline & positive cells percentage & & & & \\
\hline & $25 \%$ positive cells & 4 & Random & $1.48(0.48-4.56)$ & $<0.001$ \\
\hline & Non- $25 \%$ positive cells & 3 & Random & $1.43(0.60-3.41)$ & $<0.001$ \\
\hline \multirow[t]{7}{*}{ DFS } & ALL & 8 & Random & $2.17(1.03-4.59)$ & $<0.001$ \\
\hline & Ethnicity & & & & \\
\hline & Asian & 6 & Fixed & $3.40(2.17-5.33)$ & 0.336 \\
\hline & Caucasian & 2 & Random & $0.39(0.04-3.64)$ & 0.002 \\
\hline & Resource & & & & \\
\hline & Univariate & 4 & Random & $1.95(0.92-4.13)$ & 0.012 \\
\hline & Multivariate & 4 & Random & $2.24(0.35-14.34)$ & $<0.001$ \\
\hline PFS & ALL & 4 & Random & $1.09(0.39-3.04)$ & 0.015 \\
\hline RFS & ALL & 6 & Random & $0.93(0.64-1.35)$ & 0.013 \\
\hline
\end{tabular}

HR: hazard ratio; OS: overall survival; DFS: disease-free survival; PFS: progression-free survival; RFS: recurrence-free survival.
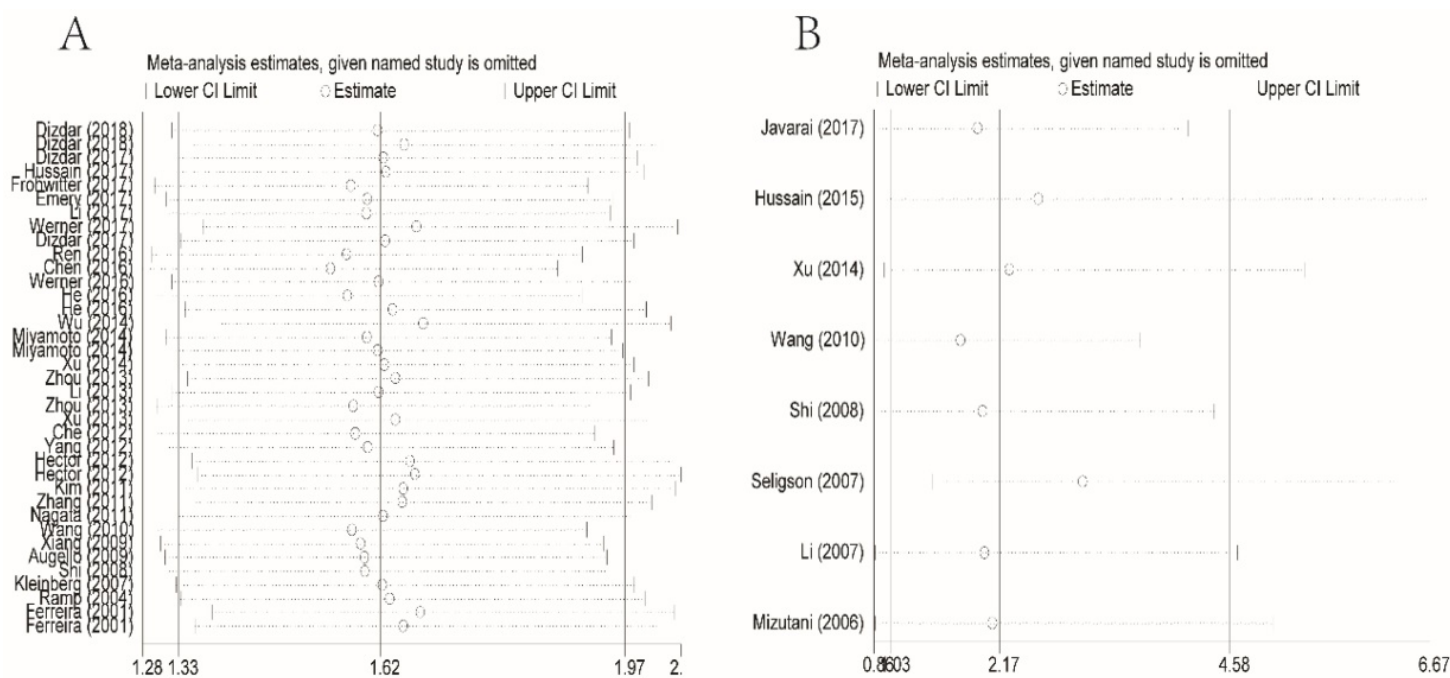

Figure 4. Effect of individual studies on the combined HR dealt with XIAP expression for OS (A) and DFS (B). HR: hazard ratio; XIAP: X-linked inhibitor of apoptosis protein; OS: overall survival; DFS: disease-free survival. 

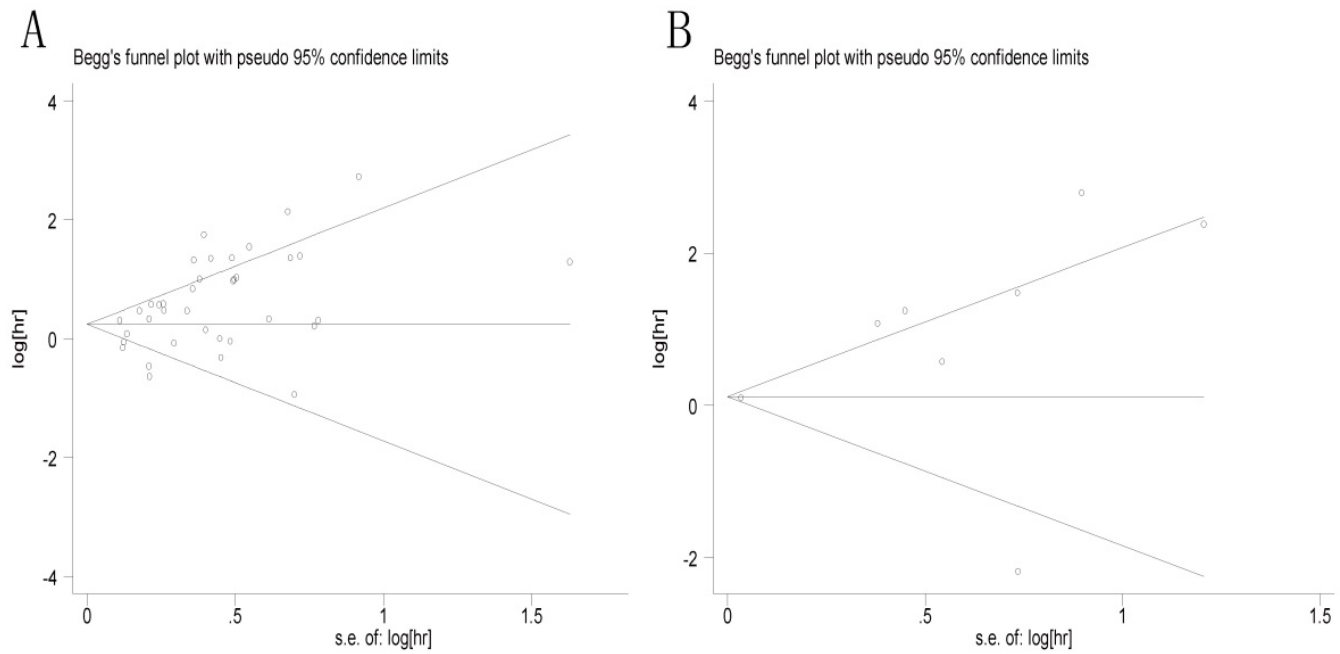

Figure 5. Begg's funnel plots for all of the included studies reported with XIAP expression for OS (A) and DFS (B). XIAP: X-linked inhibitor of apoptosis protein; OS: overall survival; DFS: disease-free survival.

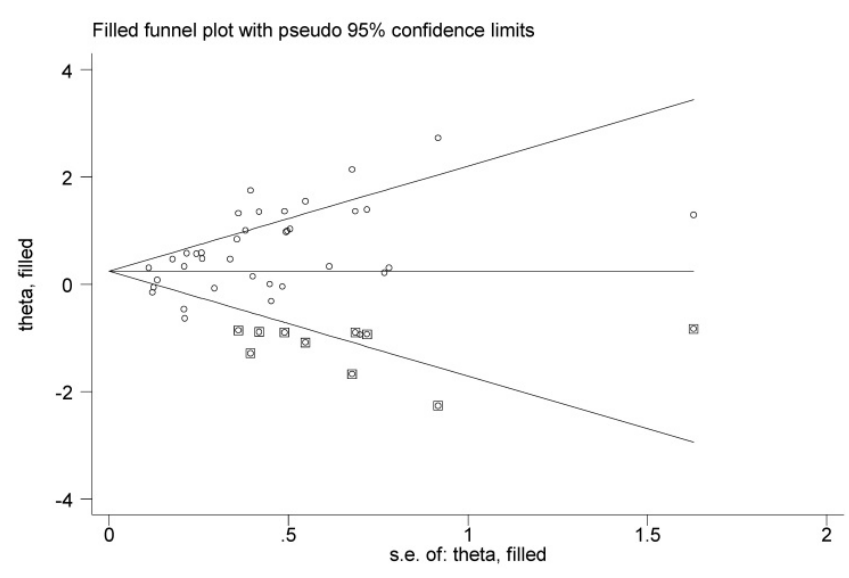

Figure 6. Funnel plot adjusted with trim and fill method. Circles: included studies; Diamonds: presumed missing studies.

\section{Discussion}

Apoptosis is a crucial procedure which plays a significant role in the preservation of tissue homeostasis and morphogenesis. The suppression of genetically encoded cell apoptosis gives rise to increased risk of carcinogenesis followed by aberrantly cell-cycle progression or enhanced accumulation of transforming mutations. In terminal stages of cancer, resistance to apoptosis is an important factor contributing to the failure of chemotherapy and radiation therapy. XIAP, which regulates caspase-dependent apoptosis at multiple levels, has been found overexpressed in many types of human malignancies. Although most studies demonstrated that expression of XIAP was associated with poor prognosis in cancer, some researchers reported contradictory predictive value of XIAP or even found no significant relationship between its level and clinical survival. Due to the inconsistency, we performed a meta-analysis to summarize the existing evidence of prognostic role of XIAP in patients with malignant tumors.

In this meta-analysis, we included a total of 40 articles with 6554 patients. Our results suggested that over-expression of XIAP correlated with poor OS and DFS of cancer patients. However, no positive association were found between XIAP detection and the PFS or RFS outcome. The insignificant results might due to the limited number of studies and discrepant source of cancer. In fact, many molecular mechanism researches have been conducted on XIAP in vitro and in vivo to date. Hofmann and his colleagues indicated that XIAP might participate in the pathogenesis of NSCLC [56]. Additionally, Berezovskaya et al. demonstrated that XIAP played a vital role in anoikis resistance and tumor metastasis in prostate cancer [57]. The majority of studies revealed that high XIAP level in cancer patients predicted poor prognosis or low survival rates. Recently, XIAP has been proposed as an attractive target for new anti-cancer interventions. Embelin, extracted from the fruits of Embelia ribes, was reported an important natural XIAP inhibitor used for clinic [58]. It binds to the BIR3 domain of XIAP, blocking the interaction of XIAP with caspase-9 to promote apoptosis and modulates anti-apoptotic pathways by suppressing the activity of NF-kB, PI3-kinase/AKT and JAK/ STAT pathway [59]. Furthermore, some smallmolecule XIAP inhibitors, like second mitochondrial activator of caspases (Smac) mimetics, have been shown to inhibit the growth of human cancer cells with little toxicity to normal tissues [60, 61]. Currently, the medicinal value of targeting XIAP in controlling cancer development and enhancing chemotherapy sensitivity has been reported in various cancer types $[10,59]$. 
As for OS, subgroup analyses revealed that unfavorable outcomes could be found in both Asian and Caucasian patients regardless of participants number, which furtherly validated the prognostic role of XIAP in cancer. The combined HR in multivariate data group was slightly higher compared to that in univariate group, suggesting that multivariate analysis method seemed to provide more reliable data. Besides, we found that high or low cut-off value set based on IHC scores did not change the positive result. However, it showed no significance when cut-off value was set by percentage of positive staining cells, implying IHC score might act as superior performance in differentiating high or low expression of XIAP. As for DFS, the outcomes of subgroup analyses exhibited a bit different from OS. Although some of results showed no statistical significance, we did not allow a definite unfavorable conclusion to its value due to limited study inclusion for the present analyses. What's more, in 40 eligible literatures, two studies focused on the XIAP detection in nucleus rather than just confined in cytoplasm_[19, 39]. But conflicting results have appeared when Zhang et al. found that XIAP positive nuclear labeling was the apoptotic marker correlated with patients' shortened OS while the study performed by Xu et al. showed no statistical significance[19, 39]. Considering few relevant articles have been published in this field, we have difficulty to draw a unanimous conclusion. In the future, more convictive studies are necessary for exploring the relationship of XIAP location to patient survival.

In addition, we performed the subgroup analyses by cancer type to further explore the specific role of XIAP. The combined HRs revealed that over-expression of XIAP predicted unfavorable OS in gastric cancer and HNC. However, this significant relationship was not found between XIAP expression and other cancer, including breast cancer, thyroid cancer and other types of digestive tumors. As one of the most surprising finding of our meta-analysis, the NSCLC patients that expressed higher levels of XIAP achieved a significantly longer OS compared with patients having lower expression of XIAP. Discrepancy among our results suggested a different role for XIAP, which might depend on the type of cancer. Then we retrieved relevant papers to explain the consequence. Tanaka et al. pointed that tumor growth is a net effect of cell death and proliferation, processes that occur simultaneously [62]. While most of the studies for XIAP only focused on apoptosis but neglected the effect of cell proliferation on tumorigenesis. To gain further insight into the role of XIAP in NSCLC patients, Ferreira analyzed tumor proliferation by assessing fraction of proliferative cells
(PI) as well as mitotic index (MI) and compared it with XIAP expression. Finally, he concluded that higher XIAP level correlated with lower tumor proliferation which might account for its relationship with a positive prognosis [40]. Similarly, another two studies revealed that XIAP expression positively associated with a favorable prognosis in prostate cancer [14, 48]. Some authors have argued that the anti-apoptotic role of XIAP are based on its overexpression in cell lines and it functions as an antior pro-apoptotic factor depending on the scenario [63]. But the molecular mechanism accounting for its possible anti-tumor role in patients still remain unclear. Remarkably, our results were strikingly similar to studies focusing on another important IAPs family member survivin, which modulated cell proliferation, procedural apoptosis and resistance of tumor cells to chemotherapy or radiotherapy just like XIAP. In previous studies, overexpressed survivin has been described correlated with poor prognosis in many tumor types like gastric cancer, renal cell carcinoma, esophageal cancer and colorectal cancer [64-67], while in pancreatic ductal adenocarcinoma, it seemed to predict a favorable clinical survival [68]. To our knowledge, this is the first meta-analysis systematically evaluating prognostic value of XIAP in different types of tumor. However, the numbers of included studies for each cancer type were limited, reducing the persuasive power of statistical data to some extent. Some of studies did not provide direct sufficient HRs so that we had to extrapolate from the survival curves, which also resulted in certain inaccuracy. Thus, we expected additional metaanalyses with a larger sample size to confirm our results in the future.

Via conducting meta-regression, we found ethnicity could explain $11.8 \%$ of the heterogeneity for studies evaluating OS. While this type of heterogeneity was difficult to exclude due to lacking sufficient patients from different backgrounds. Besides, there were several shortcomings in this meta-analysis. First, the studies retrieved were limited in articles published in English, which might partially contribute to publication bias although the result did not change after recalculating by trim and fill method. Second, we focused primarily on the prognostic significance of cancer type in this review but did not attach importance to the discrepancy among neoplasms histologic subtypes simultaneously. Moreover, other genes interacted with XIAP expression may also have some effect on patient survival, and the different cut-off values set for XIAP level both probably influence our results. We suggested that our preliminary findings warrant further analyses in the future. 


\section{Conclusion}

The results of this meta-analysis revealed significant correlations between XIAP protein level and survival of cancer patients. In subgroup analyses, over-expression of XIAP was found correlated with poor prognosis in gastric cancer and $\mathrm{HNC}$, while in NSCLC, we concluded it might serve as a favorable predictor for clinical outcomes. Given the publication bias observed across studies, we preformed trim and fill method to recalculate the data. As a result, the outcomes of us remained consistent before or after adjustment. However, due to the limited study number and significant heterogeneity, large scale researches with more complete cancer types are warranted to further verify our results.

\section{Acknowledgments}

This study was financially supported by the National Key Research and Development Program of China (ZDZX2017ZL-01), High-level innovation team of Nanjing Medical University (JX102GSP201727), Wu Jieping Foundation (320.6750.17006), Key medical talents (ZDRCA2016023), 333 Project of Jiangsu Province (BRA2017534 and BRA2015470), The collaborative innovation center for tumor individualization focuses on open topics (JX21817902/008) and Project of China key research and development program precision medicine research (2016YFC0905901).

\section{Author's Contribution}

YM.Y: Protocol/project development; X.G, J.L, H.W: Data collection or management; Y.W, YQ.Y: Data analysis; X.G, L.Z: Manuscript writing/editing.

\section{Competing Interests}

The authors have declared that no competing interest exists.

\section{References}

1. Hanahan D, Weinberg RA. Hallmarks of cancer: the next generation. Cell. 2011; 144: 646-74.

2. Galluzzi L, Vitale I, Abrams JM, Alnemri ES, Baehrecke EH, Blagosklonny MV, et al. Molecular definitions of cell death subroutines: recommendations of the Nomenclature Committee on Cell Death 2012. Cell death and differentiation. 2012; 19: 107-20.

3. de Almagro MC, Vucic D. The inhibitor of apoptosis (IAP) proteins are critical regulators of signaling pathways and targets for anti-cancer therapy. Experimental oncology. 2012; 34: 200-11.

4. Ghobrial IM, Witzig TE, Adjei AA. Targeting apoptosis pathways in cancer therapy. CA: a cancer journal for clinicians. 2005; 55: 178-94.

5. Liston P, Roy N, Tamai K, Lefebvre C, Baird S, Cherton-Horvat G, et al. Suppression of apoptosis in mammalian cells by NAIP and a related family of IAP genes. Nature. 1996; 379: 349-53.

6. Lu M, Lin SC, Huang Y, Kang YJ, Rich R, Lo YC, et al. XIAP induces NF-kappaB activation via the BIR1/TAB1 interaction and BIR1 dimerization. Molecular cell. 2007; 26: 689-702.

7. Shiozaki EN, Chai J, Rigotti DJ, Riedl SJ, Li P, Srinivasula SM, et al. Mechanism of XIAP-mediated inhibition of caspase-9. Molecular cell. 2003; 11: 519-27.

8. Riedl SJ, Renatus M, Schwarzenbacher R, Zhou Q, Sun C, Fesik SW, et al. Structural basis for the inhibition of caspase-3 by XIAP. Cell. 2001; 104: 791-800.

9. Chai J, Shiozaki E, Srinivasula SM, Wu Q, Datta P, Alnemri ES, et al. Structural basis of caspase-7 inhibition by XIAP. Cell. 2001; 104: 769-80.
10. Obexer P, Ausserlechner MJ. X-linked inhibitor of apoptosis protein - a critical death resistance regulator and therapeutic target for personalized cancer therapy. Frontiers in oncology. 2014; 4: 197.

11. Dizdar L, Junemann LM, Werner TA, Verde PE, Baldus SE, Stoecklein NH, et al. Clinicopathological and functional implications of the inhibitor of apoptosis proteins survivin and XIAP in esophageal cancer. Oncology letters. 2018; 15: 3779-89.

12. Shi YH, Ding WX, Zhou J, He JY, Xu Y, Gambotto AA, et al. Expression of $\mathrm{X}$-linked inhibitor-of-apoptosis protein in hepatocellular carcinoma promotes metastasis and tumor recurrence. Hepatology. 2008; 48: 497-507.

13. Ferreira CG, van der Valk P, Span SW, Jonker JM, Postmus PE, Kruyt FA, et al. Assessment of IAP (inhibitor of apoptosis) proteins as predictors of response to chemotherapy in advanced non-small-cell lung cancer patients. Annals of oncology : official journal of the European Society for Medical Oncology. 2001; 12: 799-805.

14. Seligson DB, Hongo F, Huerta-Yepez S, Mizutani Y, Miki T, Yu H, et al. Expression of $\mathrm{X}$-linked inhibitor of apoptosis protein is a strong predictor of human prostate cancer recurrence. Clinical cancer research : an official journal of the American Association for Cancer Research. 2007; 13: 6056-63.

15. Yim JH, Kim WG, Jeon MJ, Han JM, Kim TY, Yoon JH, et al. Association between expression of $\mathrm{X}$-linked inhibitor of apoptosis protein and the clinical outcome in a BRAF V600E-prevalent papillary thyroid cancer population. Thyroid : official journal of the American Thyroid Association. 2014; 24: 689-94.

16. Wang J, Liu Y, Ji R, Gu Q, Zhao X, Liu Y, et al. Prognostic value of the X-linked inhibitor of apoptosis protein for invasive ductal breast cancer with triple-negative phenotype. Human pathology. 2010; 41: 1186-95.

17. Wu WY, Kim H, Zhang CL, Meng XL, Wu ZS. Clinical significance of autophagic protein LC3 levels and its correlation with XIAP expression in hepatocellular carcinoma. Medical oncology. 2014; 31: 108.

18. Hussain AR, Siraj AK, Ahmed M, Bu R, Pratheeshkumar P, Alrashed AM, et al. XIAP over-expression is an independent poor prognostic marker in Middle Eastern breast cancer and can be targeted to induce efficient apoptosis. BMC cancer. 2017; 17: 640 .

19. Xu YC, Liu Q, Dai JQ, Yin ZQ, Tang L, Ma Y, et al. Tissue microarray analysis of X-linked inhibitor of apoptosis (XIAP) expression in breast cancer patients. Medical oncology. 2014; 31: 764

20. Stroup DF, Berlin JA, Morton SC, Olkin I, Williamson GD, Rennie D, et al. Meta-analysis of observational studies in epidemiology: a proposal for reporting. Meta-analysis Of Observational Studies in Epidemiology (MOOSE) group. Jama. 2000; 283: 2008-12.

21. Stang A. Critical evaluation of the Newcastle-Ottawa scale for the assessment of the quality of nonrandomized studies in meta-analyses. European journal of epidemiology. 2010; 25: 603-5.

22. Tierney JF, Stewart LA, Ghersi D, Burdett S, Sydes MR. Practical methods for incorporating summary time-to-event data into meta-analysis. Trials. 2007; 8: 16.

23. Mantel N, Haenszel W. Statistical aspects of the analysis of data from retrospective studies of disease. Journal of the National Cancer Institute. 1959; 22: $719-48$

24. DerSimonian R, Laird N. Meta-analysis in clinical trials. Controlled clinical trials. $1986: 7: 177-88$

25. Dizdar L, Tomczak M, Werner TA, Safi SA, Riemer JC, Verde PE, et al. Survivin and XIAP expression in distinct tumor compartments of surgically resected gastric cancer: XIAP as a prognostic marker in diffuse and mixed type adenocarcinomas. Oncology letters. 2017; 14: 6847-56.

26. Dizdar L, Oesterwind KA, Riemer JC, Werner TA, Mersch S, Mohlendick B, et al. Preclinical assesement of survivin and XIAP as prognostic biomarkers and therapeutic targets in gastroenteropancreatic neuroendocrine neoplasia. Oncotarget. 2017; 8: 8369-82.

27. He L, Ye F, Qu L, Wang D, Cui M, Wei C, et al. Protein profiling of alpha-fetoprotein producing gastric adenocarcinoma. Oncotarget. 2016; 7: 28448-59.

28. Kim MA, Lee HE, Lee HS, Yang HK, Kim WH. Expression of apoptosis-related proteins and its clinical implication in surgically resected gastric carcinoma. Virchows Archiv : an international journal of pathology. 2011; 459: 503-10.

29. Chen YT, Tsao SC, Tsai HP, Wang JY, Chai CY. The X-linked inhibitor of apoptosis protein is an independent prognostic marker for rectal adenocarcinoma after preoperative chemoradiotherapy. Virchows Archiv : an international journal of pathology. 2016; 468: 559-67.

30. Hector S, Conlon S, Schmid J, Dicker P, Cummins RJ, Concannon CG, et al. Apoptosome-dependent caspase activation proteins as prognostic markers in Stage II and III colorectal cancer. British journal of cancer. 2012; 106: 1499-505.

31. Xiang G, Wen X, Wang H, Chen K, Liu H. Expression of X-linked inhibitor of apoptosis protein in human colorectal cancer and its correlation with prognosis. Journal of surgical oncology. 2009; 100: 708-12

32. Li Z, Han C, Feng J. Relationship of the expression levels of XIAP and p53 genes in hepatocellular carcinoma and the prognosis of patients. Oncology letters. 2017; 14: 4037-42.

33. Che Y, Ye F, Xu R, Qing H, Wang X, Yin F, et al. Co-expression of XIAP and cyclin D1 complex correlates with a poor prognosis in patients with hepatocellular carcinoma. The American journal of pathology. 2012; 180: 1798-807. 
34. Augello C, Caruso L, Maggioni M, Donadon M, Montorsi M, Santambrogio R, et al. Inhibitors of apoptosis proteins (IAPs) expression and their prognostic significance in hepatocellular carcinoma. BMC cancer. 2009; 9: 125.

35. Zhou S, Ye W, Shao Q, Qi Y, Zhang M, Liang J. Prognostic significance of XIAP and NF-kappaB expression in esophageal carcinoma with postoperative radiotherapy. World journal of surgical oncology. 2013; 11: 288.

36. Xu JW, Zhou F, Jiang GX, Chen CY, Wang JJ, Cao LP. [Expression of pGSK-3alpha/beta Tyr279/216 and XIAP proteins in cholangiocarcinoma and their clinical significance]. Zhonghua zhong liu za zhi [Chinese journal of oncology]. 2013; 35: 366-71.

37. Zhou F, Xu J, Ding G, Cao L. Overexpressions of CK2beta and XIAP are associated with poor prognosis of patients with cholangiocarcinoma. Pathology oncology research : POR. 2014; 20: 73-9.

38. Li S, Sun J, Yang J, Zhang L, Wang L, Wang X, et al. XIAP expression is associated with pancreatic carcinoma outcome. Molecular and clinical oncology. 2013; 1: 305-8.

39. Zhang Y, Zhu J, Tang Y, Li F, Zhou H, Peng B, et al. X-linked inhibitor of apoptosis positive nuclear labeling: a new independent prognostic biomarker of breast invasive ductal carcinoma. Diagnostic pathology. 2011; 6: 49.

40. Ferreira CG, van der Valk P, Span SW, Ludwig I, Smit EF, Kruyt FA, et al. Expression of X-linked inhibitor of apoptosis as a novel prognostic marker in radically resected non-small cell lung cancer patients. Clinical cancer research : an official journal of the American Association for Cancer Research. 2001; 7: 2468-74

41. Frohwitter G, Buerger H, Korsching E, van Diest PJ, Kleinheinz J, Fillies T. Site-specific gene expression patterns in oral cancer. Head \& face medicine. 2017; 13: 6.

42. Ren M, Wang Z, Gao G, Gu X, Wu L, Chen L. Impact of X-linked inhibitor of apoptosis protein on survival of nasopharyngeal carcinoma patients following radiotherapy. Tumour biology : the journal of the International Society for Oncodevelopmental Biology and Medicine. 2016; 37: 11825-33.

43. Yang XH, Feng ZE, Yan M, Hanada S, Zuo H, Yang CZ, et al. XIAP is a predictor of cisplatin-based chemotherapy response and prognosis for patients with advanced head and neck cancer. PloS one. 2012; 7: e31601.

44. Nagata M, Nakayama H, Tanaka T, Yoshida R, Yoshitake Y, Fukuma D, et al. Overexpression of cIAP2 contributes to 5-FU resistance and a poor prognosis in oral squamous cell carcinoma. British journal of cancer. 2011; 105: 1322-30.

45. Werner TA, Dizdar L, Nolten I, Riemer JC, Mersch S, Schutte SC, et al. Survivin and XIAP - two potential biological targets in follicular thyroid carcinoma. Scientific reports. 2017; 7: 11383.

46. Werner TA, Tamkan-Olcek Y, Dizdar L, Riemer JC, Wolf A, Cupisti K, et al. Survivin and XIAP: two valuable biomarkers in medullary thyroid carcinoma. British journal of cancer. 2016; 114: 427-34.

47. Hussain AR, Bu R, Ahmed M, Jehan Z, Beg S, Al-Sobhi S, et al. Role of $\mathrm{X}$-Linked Inhibitor of Apoptosis as a Prognostic Marker and Therapeutic Target in Papillary Thyroid Carcinoma. The Journal of clinical endocrinology and metabolism. 2015; 100: E974-85.

48. Rodriguez-Berriguete $G$, Torrealba $N$, Ortega MA, Martinez-Onsurbe $P$ Olmedilla G, Paniagua R, et al. Prognostic value of inhibitors of apoptosis proteins (IAPs) and caspases in prostate cancer: caspase- 3 forms and XIAP predict biochemical progression after radical prostatectomy. BMC cancer. 2015; 15: 809.

49. Mizutani Y, Nakanishi H, Li YN, Matsubara H, Yamamoto K, Sato N, et al. Overexpression of XIAP expression in renal cell carcinoma predicts a worse prognosis. International journal of oncology. 2007; 30: 919-25.

50. Ramp U, Krieg T, Caliskan E, Mahotka C, Ebert T, Willers R, et al. XIAP expression is an independent prognostic marker in clear-cell renal carcinomas. Human pathology. 2004; 35: 1022-8.

51. Jayaraj P, Sen S, Dhanaraj PS, Jhaihria R, Singh S, Singh VK Immunohistochemical expression of X-linked inhibitor of apoptosis in eyelid sebaceous gland carcinoma predicts a worse prognosis. Indian journal of ophthalmology. 2017; 65: 1109-13.

52. Emery IF, Gopalan A, Wood S, Chow KH, Battelli C, George J, et al. Expression and function of ABCG2 and XIAP in glioblastomas. Journal of neuro-oncology. 2017; 133: 47-57.

53. Miyamoto M, Takano M, Iwaya K, Shinomiya N, Kato M, Aoyama T, et al. X-chromosome-linked inhibitor of apoptosis as a key factor for chemoresistance in clear cell carcinoma of the ovary. British journal of cancer. 2014; 110: 2881-6.

54. Li M, Song T, Yin ZF, Na YQ. XIAP as a prognostic marker of early recurrence of nonmuscular invasive bladder cancer. Chinese medical journal. 2007; 120: 469-73.

55. Kleinberg L, Lie AK, Florenes VA, Nesland JM, Davidson B. Expression of inhibitor-of-apoptosis protein family members in malignant mesothelioma. Human pathology. 2007; 38: 986-94.

56. Hofmann HS, Simm A, Hammer A, Silber RE, Bartling B. Expression of inhibitors of apoptosis (IAP) proteins in non-small cell human lung cancer. Journal of cancer research and clinical oncology. 2002; 128: 554-60.

57. Berezovskaya O, Schimmer AD, Glinskii AB, Pinilla C, Hoffman RM, Reed JC, et al. Increased expression of apoptosis inhibitor protein XIAP contributes to anoikis resistance of circulating human prostate cancer metastasis precursor cells. Cancer research. 2005; 65: 2378-86.

58. Lu H, Wang J, Wang Y, Qiao L, Zhou Y. Embelin and Its Role in Chronic Diseases. Advances in experimental medicine and biology. 2016; 928: 397-418.
59. Prabhu KS, Achkar IW, Kuttikrishnan S, Akhtar S, Khan AQ Siveen KS, et al. Embelin: a benzoquinone possesses therapeutic potential for the treatment of human cancer. Future medicinal chemistry. 2018; 10: 961-76.

60. Fulda S. Promises and Challenges of Smac Mimetics as Cancer Therapeutics. Clinical cancer research : an official journal of the American Association for Cancer Research. 2015; 21: 5030-6.

61. Sun H, Lu J, Liu L, Yi H, Oiu S, Yang CY, et al. Nonpeptidic and potent small-molecule inhibitors of cIAP-1/2 and XIAP proteins. Journal of medicinal chemistry. 2010; 53: 6361-7.

62. Tanaka F, Kawano Y, Li M, Takata T, Miyahara R, Yanagihara K, et al. Prognostic significance of apoptotic index in completely resected non-small-cell lung cancer. Journal of clinical oncology : official journal of the American Society of Clinical Oncology. 1999; 17: 2728-36.

63. Kashkar H. X-linked inhibitor of apoptosis: a chemoresistance factor or a hollow promise. Clinical cancer research : an official journal of the American Association for Cancer Research. 2010; 16: 4496-502.

64. Xiong C, Liu H, Chen Z, Yu Y, Liang C. Prognostic role of survivin in renal cell carcinoma: A system review and meta-analysis. European journal of internal medicine. 2016; 33: 102-7.

65. Xia H, Chen S, Huang H, Ma H. Survivin over-expression is correlated with a poor prognosis in esophageal cancer patients. Clinica chimica acta; international journal of clinical chemistry. 2015; 446: 82-5.

66. Krieg A, Baseras B, Tomczak M, Verde PE, Stoecklein NH, Knoefel WT. Role of survivin as prognostic and clinicopathological marker in gastric cancer: a meta-analysis. Molecular biology reports. 2013; 40: 5501-11.

67. Krieg A, Werner TA, Verde PE, Stoecklein NH, Knoefel WT. Prognostic and clinicopathological significance of survivin in colorectal cancer: a meta-analysis. PloS one. 2013; 8: e65338.

68. Jamieson NB, Carter CR, McKay CJ, Oien KA. Tissue biomarkers for prognosis in pancreatic ductal adenocarcinoma: a systematic review and meta-analysis. Clinical cancer research : an official journal of the American Association for Cancer Research. 2011; 17: 3316-31. 